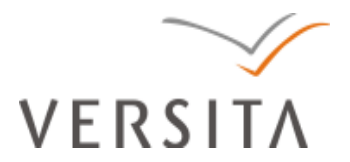

Folia Oeconomica Stetinensia

DOI: $10.2478 / \mathrm{v} 10031-010-0006-6$

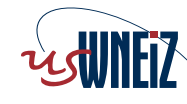

Wydzial Nauk Ekonomicznych i Zarządzania Uniwersytetu Szczecińskiego

\title{
CURVE EXTRAPOLATION AND DATA ANALYSIS USING \\ THE METHOD OF HURWITZ-RADON MATRICES
}

Dariusz Jakóbczak, Ph.D.

Department of Electronics and Computer Science

Technical University of Koszalin

Sniadeckich 2, 75-453 Koszalin, Poland

email: djakob@ie.tu.koszalin.pl

Received 24 July 2011, Accepted 29 July 2011

\begin{abstract}
Data analysis needs suitable methods of curve extrapolation. The proposed method of Hurwitz-Radon Matrices (MHR) can be used in extrapolation and interpolation of curves in the plane. For example, quotations from the Stock Exchange, the market prices or currency rates form a curve. This paper presents the way of data anticipation and extrapolation via the MHR method and decision making: to buy or not, to sell or not. The proposed method is based on a family of Hurwitz-Radon (HR) matrices. The matrices are skewsymmetric and possess columns composed of orthogonal vectors. The operator of Hurwitz-Radon (OHR), built from these matrices, is described. Two-dimensional data are represented by the set of curve points. It is shown how to create the orthogonal and discrete OHR and how to use it in a process of data foreseeing and extrapolation. The MHR method interpolates and extrapolates the curve point by point without using any formula or function.
\end{abstract}

Keywords: data analysis, decision making, curve interpolation, data extrapolation, value anticipation, the Hurwitz-Radon matrices.

JEL classification: $\mathrm{C} 02$. 


\section{Introduction}

A significant problem in data analysis and artificial intelligence ${ }^{1}$ is that of appropriate data representation and extrapolation. Two-dimensional data can be treated as points on the curve. Classical polynomial interpolation or extrapolation (Lagrange, Newton, Hermite) is useless for data anticipation, because the stock quotations or the market prices represent discrete data and they do not preserve a shape of the polynomial. Also Richardson extrapolation has some weak sides concerning discrete data. This paper is dealing with the method of value foreseeing by using a family of Hurwitz-Radon matrices. The quotations, prices or rate of a currency, represented by curve points, consist of information which allows us to extrapolate the next data and then to make a decision ${ }^{2}$.

If the probabilities of possible actions are known, then some criteria are to apply: Laplace, Bayes, Wald, Hurwicz, Savage, Hodge-Lehmann ${ }^{3}$ and others ${ }^{4}$. But author of this paper considers only two possibilities: to do something or not. For example to buy a share or not, to sell a currency or not. Proposed method of Hurwitz-Radon Matrices (MHR) is used in data extrapolation and then calculations for decision making are described. MHR method uses two-dimensional data for knowledge representation ${ }^{5}$ and computational foundations ${ }^{6}$. Also medicine ${ }^{7}$, industry and manufacturing are looking for the methods connected with geometry of the curves ${ }^{8}$. So suitable data representation and precise reconstruction or extrapolation ${ }^{9}$ of the curve is a key factor in many applications of artificial intelligence and knowledge representation.

\section{Curve and Data Representation}

Data are represented by the set of curve points $\left(x_{i}, y_{i}\right) \in \boldsymbol{R}^{2}$ (interpolation nodes) as follows in novel MHR method:

1. nodes (characteristic points) are settled at local extrema (maximum or minimum) of one of coordinates and at least one point between two successive local extrema;

2. nodes $\left(x_{i}, y_{i}\right)$ are monotonic in coordinates $x_{i}\left(x_{i}<x_{i+1}\right.$ for all $\left.i\right)$ or $y_{i}\left(y_{i}<y_{i+1}\right)$;

3. one curve is represented by at least five nodes.

Condition 1 is done for the most appropriate description of a curve. The quotations or prices are real coordinates of nodes. Condition 2 according to a graph of function means that $x_{i}$ represent for example the time. Condition 3 is adequate to interpolation, but in extrapolation minimal number of nodes is four. 


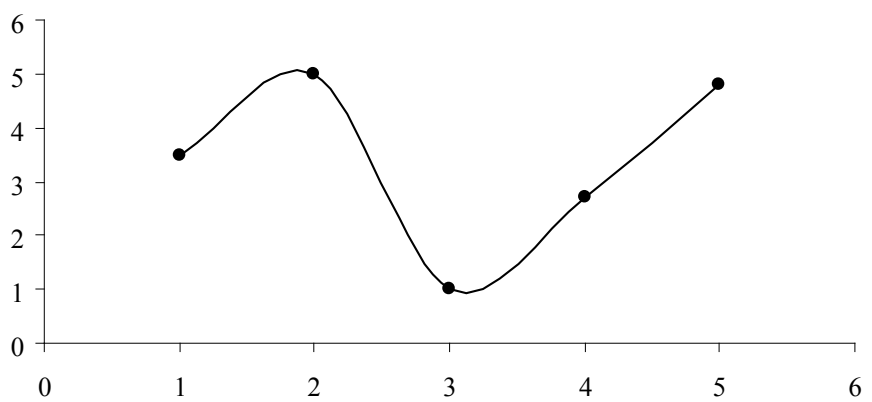

Fig. 1. Five nodes of data and the curve

Source: own study.

Data points are treated as interpolation nodes. How can we extrapolate continues values at time $x=5.5$ for example or discrete data for next day $x=6$ (Figure 1)? The anticipation of values is possible using the proposed MHR method.

\section{Curve and Data Reconstruction}

The following question is important in computer sciences and mathematics: is it possible to find a method of curve extrapolation in the plane without building the interpolation and extrapolation polynomials or other functions? This paper aims at giving the positive answer to this question. When comparing the MHR method with the Bézier curves, the Hermite curves and the B-curves (B-splines) or the NURBS one unpleasant feature of these curves must be mentioned: any small change of one characteristic point can result in a big change of the whole reconstructed curve. Such a feature does not appear in the MHR method. The methods of curve interpolation or extrapolation based on classical polynomial interpolations are: the Newton, the Lagrange or the Hermite polynomials and the spline curves which are piecewise polynomials ${ }^{10}$. Classical methods are useless to interpolate a function that fails to be differentiable at one point. Also, when the graph of interpolated or extrapolated function differs from the shape of polynomials considerably, for example $f(x)=1 / x$, interpolation and extrapolation is very hard because of existing local extrema of polynomial. The Lagrange interpolation polynomial for the function $f(x)=1 / x$ and nodes $(5 ; 0.2),(5 / 3 ; 0.6),(1 ; 1),(5 / 7 ; 1.4),(5 / 9 ; 1.8)$ has one minimum and two roots. the Lagrange interpolation polynomial differs extremely from the shape of the function $f(x)=1 / x$. 
We cannot forget about Runge's phenomenon: when the interpolation nodes are equidistance, then a high-order polynomial oscillates towards the end of the interval, for example close to -1 and 1 with the function $f(x)=1 /\left(1+25 x^{2}\right)$ and extrapolation is impossible ${ }^{11}$. The method of Hurwitz-Radon Matrices (MHR), described in this paper, is free of these undesirable examples. The curve or function in the MHR method is parameterized for a real number $\alpha \in[0 ; 1]$ in the range of two successive interpolation nodes. The MHR in data extrapolation is possible with $\alpha<0$ or $\alpha>1$.

\subsection{The Operator of Hurwitz-Radon}

Adolf Hurwitz (1859-1919) and Johann Radon (1887-1956) published the papers about a specific class of matrices in 1923 when working on the problem of quadratic forms. Matrices $A_{i}, i=1,2, \ldots, m$ satisfying:

$$
A_{j} A_{k}+A_{k} A_{j}=0, A_{j}^{2}=-I \text { for } j \neq k ; j, k=1,2, \ldots, m,
$$

are called a family of Hurwitz-Radon matrices. The family of Hurwitz-Radon (HR) matrices has important features ${ }^{12}$ : the HR matrices are skew-symmetric $\left(A_{i}^{T}=-A_{i}\right)$ and reverse matrices are easy to find $\left(A_{i}^{-1}=-A_{i}\right)$. Only for the dimension $N=2,4$ or 8 the family of HR matrices consists of $N-1$ matrices. For $N=2$ there is one matrix:

$$
A_{1}=\left[\begin{array}{cc}
0 & 1 \\
-1 & 0
\end{array}\right] \text {. }
$$

For $N=4$ there are three HR matrices with integer entries:

$$
A_{1}=\left[\begin{array}{cccc}
0 & 1 & 0 & 0 \\
-1 & 0 & 0 & 0 \\
0 & 0 & 0 & -1 \\
0 & 0 & 1 & 0
\end{array}\right], \quad A_{2}=\left[\begin{array}{cccc}
0 & 0 & 1 & 0 \\
0 & 0 & 0 & 1 \\
-1 & 0 & 0 & 0 \\
0 & -1 & 0 & 0
\end{array}\right], \quad A_{3}=\left[\begin{array}{cccc}
0 & 0 & 0 & 1 \\
0 & 0 & -1 & 0 \\
0 & 1 & 0 & 0 \\
-1 & 0 & 0 & 0
\end{array}\right] .
$$

For $N=8$ we have seven HR matrices with elements $0, \pm 1$.

So far the HR matrices are applied in electronics ${ }^{13}$ : in Space-Time Block Coding (STBC) and orthogonal design ${ }^{14}$, also in signal processing ${ }^{15}$ and Hamiltonian Neural Nets ${ }^{16}$.

If one curve is described by a set of data points $\left\{\left(x_{i}, y_{i}\right), i=1,2, \ldots, n\right\}$ monotonic in coordinates $x_{i}$ (time for example), then the HR matrices combined with the identity matrix $I_{N}$ are used to build the orthogonal and discrete Hurwitz-Radon Operator (OHR). For the nodes $\left(x_{1}, y_{1}\right)$ and $\left(x_{2}, y_{2}\right), x_{1}<x_{2}$ OHR $M$ of the dimension $N=2$ is constructed: 


$$
M=\frac{1}{x_{1}^{2}+x_{2}^{2}}\left[\begin{array}{ll}
x_{1} y_{1}+x_{2} y_{2} & x_{2} y_{1}-x_{1} y_{2} \\
x_{1} y_{2}-x_{2} y_{1} & x_{1} y_{1}+x_{2} y_{2}
\end{array}\right]
$$

For the nodes $\left(x_{1}, y_{1}\right),\left(x_{2}, y_{2}\right),\left(x_{3}, y_{3}\right)$ and $\left(x_{4}, y_{4}\right)$, monotonic in $x_{i}$, OHR of the dimension $N=4$ is constructed:

$$
M=\frac{1}{x_{1}^{2}+x_{2}^{2}+x_{3}^{2}+x_{4}^{2}}\left[\begin{array}{cccc}
u_{0} & u_{1} & u_{2} & u_{3} \\
-u_{1} & u_{0} & -u_{3} & u_{2} \\
-u_{2} & u_{3} & u_{0} & -u_{1} \\
-u_{3} & -u_{2} & u_{1} & u_{0}
\end{array}\right]
$$

where

$$
\begin{array}{cc}
u_{0}=x_{1} y_{1}+x_{2} y_{2}+x_{3} y_{3}+x_{4} y_{4}, & u_{1}=-x_{1} y_{2}+x_{2} y_{1}+x_{3} y_{4}-x_{4} y_{3}, \\
u_{2}=-x_{1} y_{3}-x_{2} y_{4}+x_{3} y_{1}+x_{4} y_{2}, & u_{3}=-x_{1} y_{4}+x_{2} y_{3}-x_{3} y_{2}+x_{4} y_{1} .
\end{array}
$$

For the nodes $\left(x_{1}, y_{1}\right),\left(x_{2}, y_{2}\right), \ldots,\left(x_{8}, y_{8}\right)$, monotonic in $x_{i}$, OHR of the dimension $N=8$ is built ${ }^{17}$ similarly as (1) and (2):

$$
M=\frac{1}{\sum_{i=1}^{8} x_{i}^{2}}\left[\begin{array}{cccccccc}
u_{0} & u_{1} & u_{2} & u_{3} & u_{4} & u_{5} & u_{6} & u_{7} \\
-u_{1} & u_{0} & u_{3} & -u_{2} & u_{5} & -u_{4} & -u_{7} & u_{6} \\
-u_{2} & -u_{3} & u_{0} & u_{1} & u_{6} & u_{7} & -u_{4} & -u_{5} \\
-u_{3} & u_{2} & -u_{1} & u_{0} & u_{7} & -u_{6} & u_{5} & -u_{4} \\
-u_{4} & -u_{5} & -u_{6} & -u_{7} & u_{0} & u_{1} & u_{2} & u_{3} \\
-u_{5} & u_{4} & -u_{7} & u_{6} & -u_{1} & u_{0} & -u_{3} & u_{2} \\
-u_{6} & u_{7} & u_{4} & -u_{5} & -u_{2} & u_{3} & u_{0} & -u_{1} \\
-u_{7} & -u_{6} & u_{5} & u_{4} & -u_{3} & -u_{2} & u_{1} & u_{0}
\end{array}\right]
$$

where

$$
u=\left[\begin{array}{cccccccc}
y_{1} & y_{2} & y_{3} & y_{4} & y_{5} & y_{6} & y_{7} & y_{8} \\
-y_{2} & y_{1} & -y_{4} & y_{3} & -y_{6} & y_{5} & y_{8} & -y_{7} \\
-y_{3} & y_{4} & y_{1} & -y_{2} & -y_{7} & -y_{8} & y_{5} & y_{6} \\
-y_{4} & -y_{3} & y_{2} & y_{1} & -y_{8} & y_{7} & -y_{6} & y_{5} \\
-y_{5} & y_{6} & y_{7} & y_{8} & y_{1} & -y_{2} & -y_{3} & -y_{4} \\
-y_{6} & -y_{5} & y_{8} & -y_{7} & y_{2} & y_{1} & y_{4} & -y_{3} \\
-y_{7} & -y_{8} & -y_{5} & y_{6} & y_{3} & -y_{4} & y_{1} & y_{2} \\
-y_{8} & y_{7} & -y_{6} & -y_{5} & y_{4} & y_{3} & -y_{2} & y_{1}
\end{array}\right] \cdot\left[\begin{array}{c}
x_{1} \\
x_{2} \\
x_{3} \\
x_{4} \\
x_{5} \\
x_{6} \\
x_{7} \\
x_{8}
\end{array}\right]
$$


The components of the vector $\mathbf{u}=\left(u_{0}, u_{1}, \ldots, u_{7}\right)^{T}$, appearing in the matrix $M(3)$, are defined by (4) in the similar way to (1)-(2) but in terms of the coordinates of the above 8 nodes. Note that the OHR operators $M(1)-(3)$ satisfy the condition of interpolation:

$$
M \cdot \mathrm{x}=\mathrm{y}
$$

for $\mathbf{x}=\left(x_{1}, x_{2}, \ldots, x_{N}\right)^{T} \in \boldsymbol{R}^{N}, \mathbf{x} \neq \mathbf{0}, \mathbf{y}=\left(y_{1}, y_{2}, \ldots, y_{N}\right)^{T} \in \boldsymbol{R}^{N}, N=2,4$ or 8 .

If one curve is described by a set of nodes $\left\{\left(x_{i}, y_{i}\right), i=1,2, \ldots, n\right\}$ monotonic in coordinates $y_{i}$, then the HR matrices combined with the identity matrix $I_{N}$ are used to build the orthogonal and discrete reverse Hurwitz-Radon Operator (reverse OHR) $M^{-1}$. If the matrix $M$ in (1)-(3) is described as:

$$
M=\frac{1}{\sum_{i=1}^{N} x_{i}^{2}}\left(u_{0} \cdot I_{N}+D\right),
$$

where the matrix $D$ consists of elements 0 (diagonal) and $u_{1}, \ldots, u_{\mathrm{N}-1}$, then the reverse OHR $M^{-1}$ is given by:

$$
M^{-1}=\frac{1}{\sum_{i=1}^{N} y_{i}^{2}}\left(u_{0} \cdot I_{N}-D\right)
$$

Note that the reverse OHR operator (6) satisfies the condition of interpolation

$$
M^{-1} \cdot y=x
$$

for $\mathbf{x}=\left(x_{1}, x_{2}, \ldots, x_{N}\right)^{\mathrm{T}} \in \boldsymbol{R}^{N}, \mathbf{y}=\left(y_{1}, y_{2}, \ldots, y_{N}\right)^{\mathrm{T}} \in \boldsymbol{R}^{N}, \mathbf{y} \neq \mathbf{0}, N=2,4$ or 8 .

\subsection{Data Extrapolation and MHR Method}

The key question is as follows: how can we compute coordinates of points settled between the interpolation nodes ${ }^{18}$ or beyond the nodes? The answer is connected with the proposed MHR method for interpolation ${ }^{19}$ and extrapolation. On a segment of a line every number " $c$ " situated between " $a$ " and " $b$ " is described by the linear (convex) combination $c=\alpha \cdot a+(1-\alpha) \cdot b$ for:

$$
\alpha=\frac{b-c}{b-a} \in[0 ; 1]
$$

If $c<a$ then $\alpha>1$ : the possible extrapolation of points is situated left of the nodes. If $c>b$ then $\alpha<0$ and the possible extrapolation of points is situated right of the nodes.

When the nodes are monotonic in the coordinates $x_{i}$, the average OHR operator $M_{2}$ of the dimension $N=2,4$ or 8 is constructed as follows:

$$
M_{2}=\alpha \cdot M_{0}+(1-\alpha) \cdot M_{1}
$$


with the operator $M_{0}$ built (1)-(3) by the "odd" nodes $\left(x_{1}=a, y_{1}\right),\left(x_{3}, y_{3}\right), \ldots,\left(x_{2 N-1}, y_{2 N-1}\right)$ and $M_{1}$ built (1)-(3) by the "even" nodes $\left(x_{2}=b, y_{2}\right),\left(x_{4}, y_{4}\right), \ldots,\left(x_{2 N}, y_{2 N}\right)$. Having the operator $M_{2}$ for the coordinates $x_{i}<x_{i+1}$ it is possible to reconstruct the second coordinates of points $(x, y)$ in terms of the vector $C$ defined with:

$$
c_{i}=\alpha \cdot x_{2 i-1}+(1-\alpha) \cdot x_{2 i}, i=1,2, \ldots, N
$$

as $C=\left[c_{1}, c_{2}, \ldots, c_{N}\right]^{T}$. The required formula is similar to (5):

$$
Y(C)=M_{2} \cdot C
$$

in which components of the vector $Y(C)$ give the second coordinate of the points $(x, y)$ corresponding to the first coordinate, given in terms of components of the vector $C$.

On the other hand, having the operator $M_{2}^{-1}$ for the coordinates $y_{i}<y_{i+1}$ it is possible to reconstruct the first coordinates of points $(x, y)$ :

$$
\begin{gathered}
M_{2}^{-1}=\alpha \cdot M_{0}^{-1}+(1-\alpha) \cdot M_{1}^{-1}, c_{i}=\alpha \cdot y_{2 i-1}+(1-\alpha) \cdot y_{2 i}, \\
X(c)=M_{2}^{-1} c
\end{gathered}
$$

Calculation of unknown coordinates for curve points using (8)-(12) is called by author the method of Hurwitz-Radon Matrices $(\mathrm{MHR})^{20}$. Here are the applications of the MHR method for the functions $f(x)=1 /\left(1+25 x^{2}\right)$ with five nodes equidistance in the first coordinate: $x_{i}=-1$, $-0.5,0,0.5,1$.

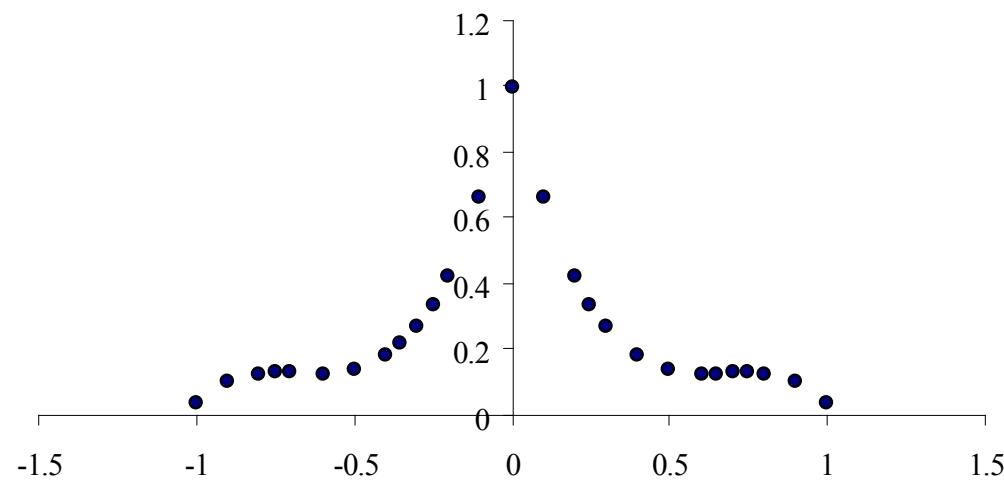

Fig. 2. 26 interpolated points of functions $f(x)=1 /\left(1+25 x^{2}\right)$ using the MHR method with 5 nodes

Source: own study. 
The MHR interpolation of the function $f(x)=1 /\left(1+25 x^{2}\right)$ gives a better result than the Lagrange interpolation. The same can be said about the function $f(x)=1 / x^{21}$.

The MHR extrapolation is valid for $\alpha<0$ or $\alpha>1$. In the case of the continuous data, the parameter $\alpha$ is a real number. For example, there are four nodes: $(1 ; 2),(1.3 ; 5),(2 ; 3),(2.5 ; 6)$. The MHR extrapolation with $\alpha=-0.01$ gives the point $(2.505 ; 6.034)$ and with $\alpha=-0.1$ : $(2.55$; 6.348). But the rate of a currency or the quotations are discrete data. If we assume that the rate of a currency is represented by equidistance nodes (day by day - fixed step of the time $h=1$ for the coordinate $x$ ), the next data or the rate on the next day is extrapolated (anticipated) with $\alpha=-1$.

\subsection{Complexity of the MHR calculations}

The MHR interpolation of a curve consists of $L$ points: if we have $n$ interpolation nodes, then there is $K=L-n$ points to find by means of the MHR method. Now let us consider the complexity of the MHR calculations.

Lemma 1. Let $n=5,9$ or 17 be the number of interpolation nodes, let the MHR method is done for the reconstruction of the curve consisting of L points. Then the MHR method is connected with the computational cost of rank $O(L)$.

Proof. Using the MHR method we have to reconstruct $K=L-n$ points of an unknown curve. Counting the number of multiplications and divisions $D$ we obtain the following results:

1) $D=4 L+7$ for $n=5$ and $L=2 i+5$;

2) $D=6 L+21$ for $n=9$ and $L=4 i+9$;

3) $D=10 L+73$ for $n=17$ and $L=8 i+17 ; \quad i=2,3,4 \ldots$

The lowest computational cost appears in the MHR method with five nodes and the OHR operators of the dimension $N=2$.

\section{Data Analysis and Decision Making}

\section{Example 1}

The MHR calculations are done for true rates of euro at the National Bank of Poland (NBP) from January $24^{\text {th }}$ to February $14^{\text {th }}, 2011$. If the last four rates are considered: $(1 ; 3.8993)$, $(2 ; 3.9248),(3 ; 3.9370)$ and $(4 ; 3.9337)$, the MHR extrapolation with matrices of the dimension $N=2$ gives the result of $(5 ; 3.9158)$. So, the anticipated rate of euro on the day February $15^{\text {th }}$ is 3.9158 (Figure 3). 


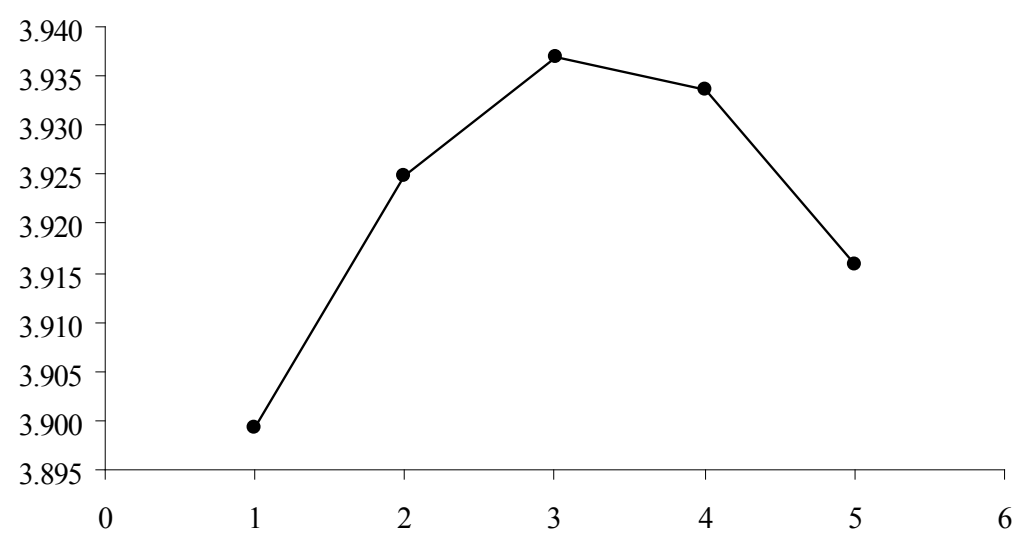

Fig. 3. The extrapolated rate for the day 5 (February $15^{\text {th }}$ ) using the MHR method with 4 nodes

Source: own study.

If the last eight rates are considered: $(1 ; 3.9173),(2 ; 3.9075),(3 ; 3.8684),(4 ; 3.8742)$, $(5 ; 3.8993),(6 ; 3.9248),(7 ; 3.9370)$ and $(8 ; 3.9337)$, the MHR extrapolation with matrices of the dimension $N=4$ gives the result of $(9 ; 4.0767)$. The anticipated rate of euro on February $15^{\text {th }}$ is 4.0767 (Figure 4).

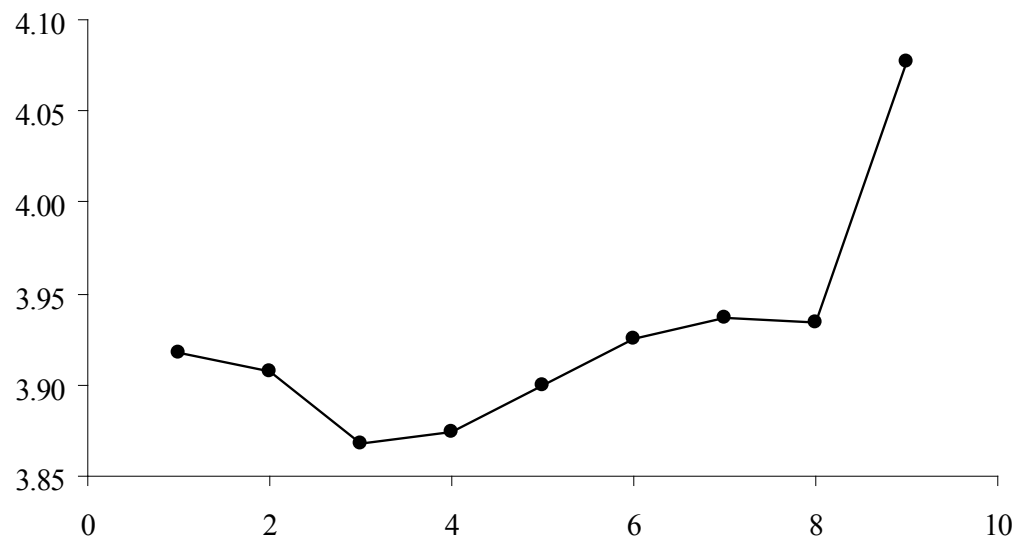

Fig. 4. The extrapolated rate for the day 9 (February $\left.15^{\text {th }}\right)$ using MHR method with 8 nodes Source: own study.

There are two extrapolated values for the next day. This example gives us two anticipated rates for tomorrow: 3.9158 and 4.0767, which differs considerably. How these extrapolated 
values can be used in the process of making a decision if to buy euro or not, to sell euro or not? The proposal final anticipated rate of euro for February $15^{\text {th }}$ (Figure 5) based on the weighted mean value:

$$
\frac{2 \cdot 3.9158+4.0767}{3}=3.9694
$$

because the rate 3.9158 is calculated for $N=2$, whereas 4.0767 is extrapolated for $N=4$. Formula (13) takes one fact into account: the dimension $N=4$ is two times bigger than the dimension $N=2$ and the result 3.9158 has to be strengthened by multiplying it by two.

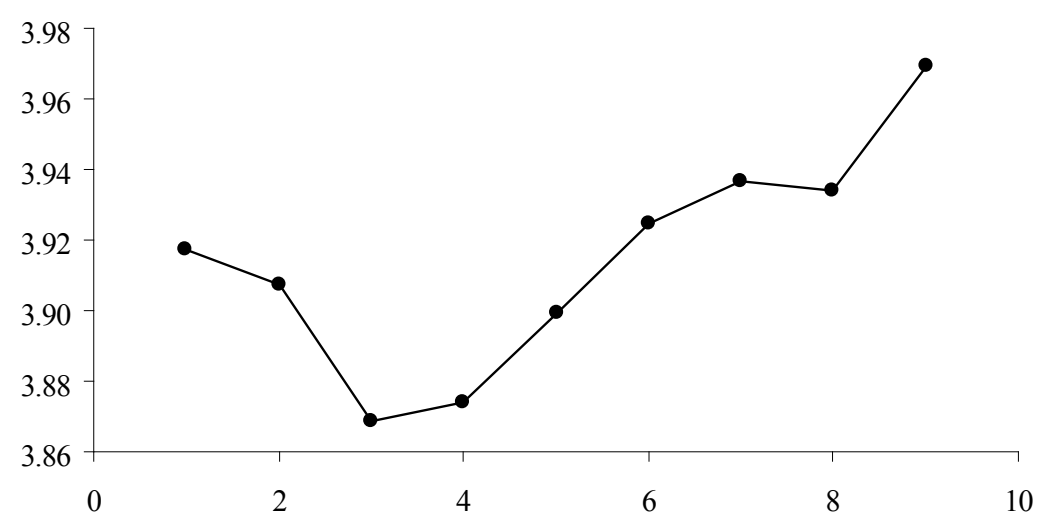

Fig. 5. The extrapolated rate for the day 9 (February $\left.15^{\text {th }}\right)$ using the MHR method with 8 nodes and the weighted mean value (13)

Source: own study.

If the last sixteen rates are taken into consideration, the MHR extrapolation with matrices of the dimension $N=8$ has to be used. Here are the rates: $(1 ; 3.8765),(2 ; 3.8777),(3 ; 3.8777)$, $(4 ; 3.9009),(5 ; 3.9111),(6 ; 3.9345),(7 ; 3.9129),(8 ; 3.9019),(9 ; 3.9173),(10 ; 3.9075)$, $(11 ; 3.8684),(12 ; 3.8742),(13 ; 3.8993),(14 ; 3.9248),(15 ; 3.9370)$ and $(16 ; 3.9337)$. The average OHR operator $M_{2}$ and the MHR calculations look as follows:

$$
M_{2}=\left[\begin{array}{cccccccc}
0.3226 & 0.0154 & 0.0286 & 0.0462 & -0.062 & 0.0444 & 0.0924 & 0.0461 \\
-0.0154 & 0.3226 & 0.0462 & -0.0286 & 0.0444 & 0.062 & -0.0461 & 0.0924 \\
-0.0286 & -0.0462 & 0.3226 & 0.0154 & 0.0924 & 0.0461 & 0.062 & -0.0444 \\
-0.0462 & 0.0286 & -0.0154 & 0.3226 & 0.0461 & -0.0924 & 0.0444 & 0.062 \\
0.062 & -0.0444 & -0.0924 & -0.0461 & 0.3226 & 0.0154 & 0.0286 & 0.0462 \\
-0.0444 & -0.062 & -0.0461 & 0.0924 & -0.0154 & 0.3226 & -0.0462 & 0.0286 \\
-0.0924 & 0.0461 & -0.062 & -0.0444 & -0.0286 & 0.0462 & 0.3226 & -0.0154 \\
-0.0461 & -0.0924 & 0.0444 & -0.062 & -0.0462 & -0.0286 & 0.0154 & 0.3226
\end{array}\right],
$$




$$
M_{2} \cdot\left[\begin{array}{c}
3 \\
5 \\
7 \\
9 \\
11 \\
13 \\
15 \\
17
\end{array}\right]=\left[\begin{array}{l}
3.7252 \\
3.8072 \\
3.8704 \\
3.8278 \\
3.8653 \\
3.8834 \\
3.9825 \\
3.9882
\end{array}\right] .
$$

The MHR extrapolation gives the result of $(17 ; 3.9882)$. The anticipated rate of euro for February $15^{\text {th }}$ is 3.9882 (Figure 6).

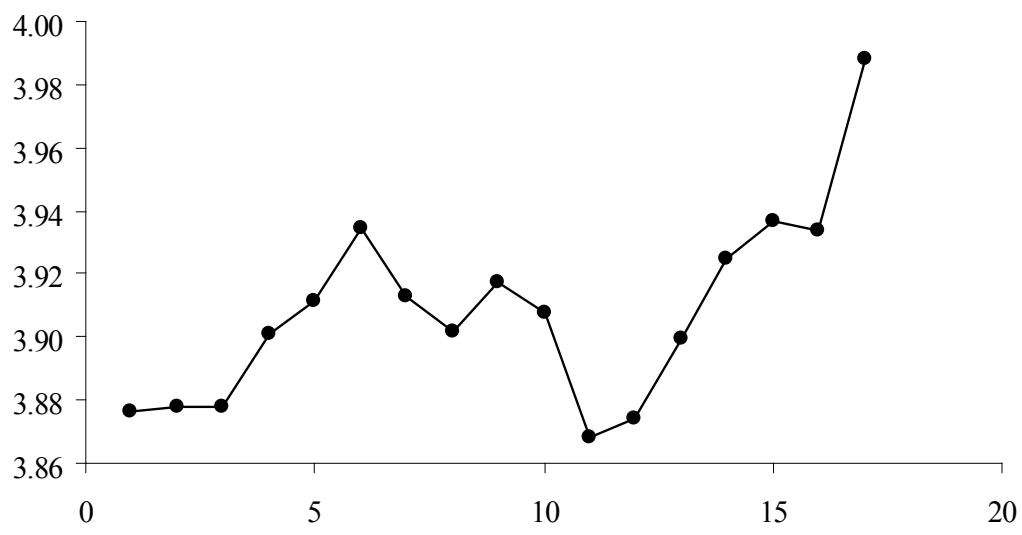

Fig. 6. The extrapolated rate for the day 17 (February $15^{\text {th }}$ ) using the MHR method with 16 nodes

Source: own study.

The MHR extrapolation has been done three times $(N=2,4$ or 8$)$ and the anticipated values are 3.9158, 4.0767 and 3.9882 respectively. The proposed final anticipated rate of euro for February $15^{\text {th }}$ (Figure 7 ) based on weighted mean value is:

$$
\frac{4 \cdot 3.9158+2 \cdot 4.0767+3.9882}{7}=3.9721
$$

because the rate of 3.9158 is calculated with the last four data points, 4.0767 is extrapolated for the last eight data points and 3.9882 is computed for the last sixteen data points. Formula (14) takes one fact into account: the number of sixteen points is four times bigger than four and two 
times bigger than eight. The result of 3.9158 has to be strengthened by multiplying it by four and the rate of 4.0767 has to be strengthened by multiplying it by two.

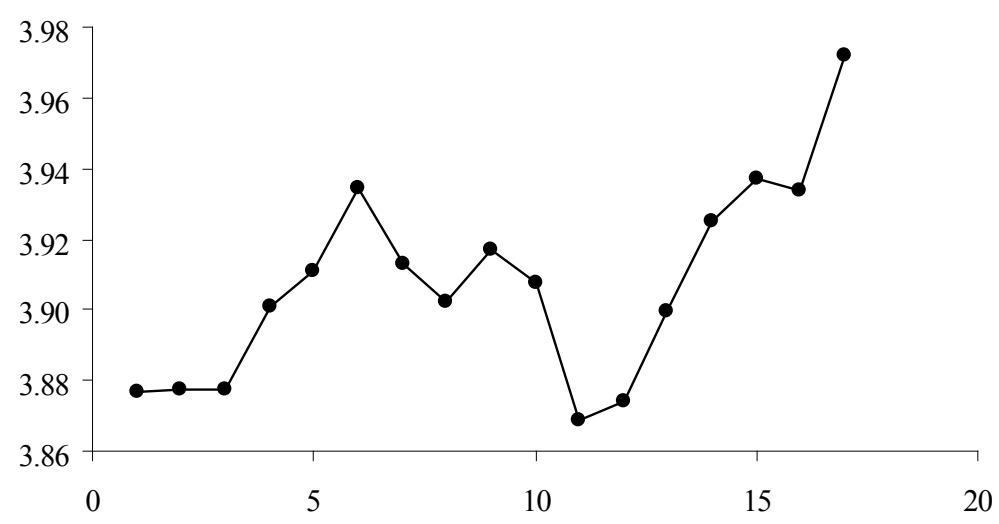

Fig. 7. The extrapolated rate for the day 17 (February $\left.15^{\text {th }}\right)$ using the MHR method with 16 nodes and the weighted mean value (14)

Source: own study.

The true rate of euro for February $15^{\text {th }}$ is 3.9398 (Figure 8).

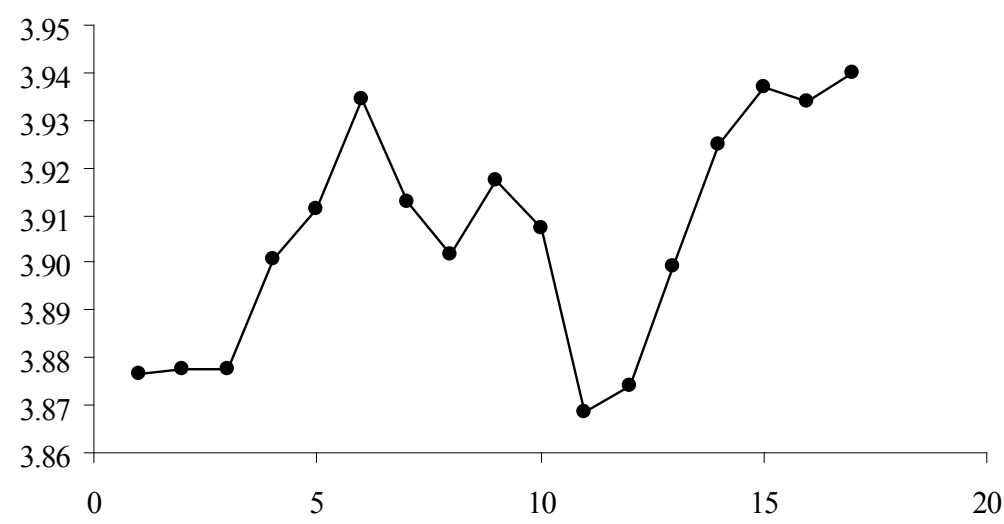

Fig. 8. The true rate of euro for the day 17 (February $15^{\text {th }}$ )

Source: own study.

In the author's opinion, values extrapolated for the next day of 3.9694 (13) and 3.9721 (14) are good enough to be one of the factors for making a decision of buying or selling the currency. 


\section{Example 2}

The MHR calculations are done for true rates of US dollar at the National Bank of Poland (NBP) from June $16^{\text {th }}$ to July $8^{\text {th }}, 2011$ (Friday). If the last four rates are considered: $(1 ; 2.7266)$, $(2 ; 2.7531),(3 ; 2.7597)$ and $(4 ; 2.7505)$, the MHR extrapolation with matrices of the dimension $N=2$ gives the result of $(5 ; 2.7239)$ :

$$
M_{2}=\left[\begin{array}{cc}
0.5503 & 0.00913 \\
-0.00913 & 0.5503
\end{array}\right], \quad M_{2} \cdot\left[\begin{array}{l}
3 \\
5
\end{array}\right]=\left[\begin{array}{c}
1.6964 \\
2.7239
\end{array}\right] \text {. }
$$

So the anticipated rate of US dollar on July $11^{\text {th }}$ (Monday) is 2.7239 .

If the last eight rates are considered: $(1 ; 2.7877),(2 ; 2.7517),(3 ; 2.7273),(4 ; 2.7156)$, $(5 ; 2.7266),(6 ; 2.7531),(7 ; 2.7597)$ and $(8 ; 2.7505)$, the MHR extrapolation with matrices of the dimension $N=4$ gives the result of $(9 ; 2.8471)$ :

$$
M_{2}=\left[\begin{array}{cccc}
0.3919 & -0.0032 & 0.0999 & 0.0527 \\
0.0032 & 0.3919 & -0.0527 & 0.0999 \\
-0.0999 & 0.0527 & 0.3919 & 0.0032 \\
-0.0527 & -0.0999 & -0.0032 & 0.3919
\end{array}\right], M_{2} \cdot\left[\begin{array}{l}
3 \\
5 \\
7 \\
9
\end{array}\right]=\left[\begin{array}{l}
2.3333 \\
2.4999 \\
2.7365 \\
2.8471
\end{array}\right] \text {. }
$$

The anticipated rate of US dollar on July $11^{\text {th }}$ is 2.8471 . There are two extrapolated values for the next day. Example 2 gives us two anticipated rates for tomorrow: 2.7239 and 2.8471 . How these extrapolated values can be used in the process of making a decision if to buy dollar

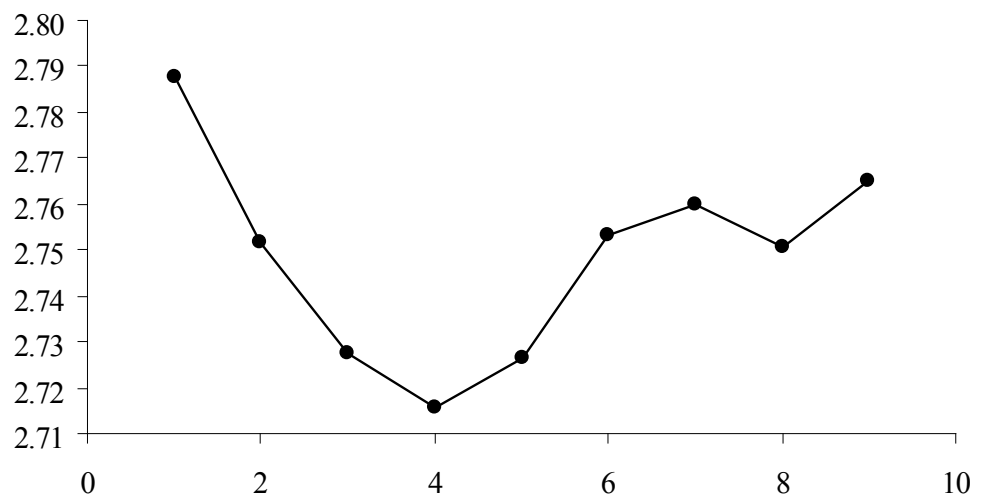

Fig. 9. The extrapolated rate for the day $9\left(\right.$ July $\left.11^{\text {th }}\right)$ using the MHR method with 8 nodes and the weighted mean value (15)

Source: own study. 
or not, to sell dollar or not? The proposed final anticipated rate of US dollar (Figure 9) on July $11^{\text {th }}$ based on weighted mean value is:

$$
\frac{2 \cdot 2.7239+2.8471}{3}=2.7650
$$

because the rate 2.7239 is calculated for $N=2$, whereas 2.8471 is extrapolated for $N=4$.

If the last sixteen rates are considered, the MHR extrapolation with matrices of the dimension $N=8$ has to be used. Here are the rates: $(1 ; 2.8069),(2 ; 2.8077),(3 ; 2.8058),(4 ; 2.7776)$, (5; 2.7661), (6; 2.7914), (7; 2.8201), (8; 2.8055), (9; 2.7877), (10; 2.7517), (11; 2.7273), $(12 ; 2.7156),(13 ; 2.7266),(14 ; 2.7531),(15 ; 2.7597)$ and $(16 ; 2.7505)$. The MHR extrapolation gives the result of $(17 ; 2.7808)$. The anticipated rate of US dollar on July $11^{\text {th }}$ is 2.7808 .

The MHR extrapolation has been done three times $(N=2,4$ or 8$)$ and the anticipated values are $2.7239,2.8471$ and 2.7808 , respectively. The proposed final anticipated rate of US dollar (Figure 10) on July $11^{\text {th }}$ based on the weighted mean value is:

$$
\frac{4 \cdot 2.7239+2 \cdot 2.8471+2.7808}{7}=2.7672
$$

because the rate of 2.7239 is calculated with the last four data points, 2.8471 is extrapolated for the last eight data points and 2.7808 is computed for the last sixteen data points.

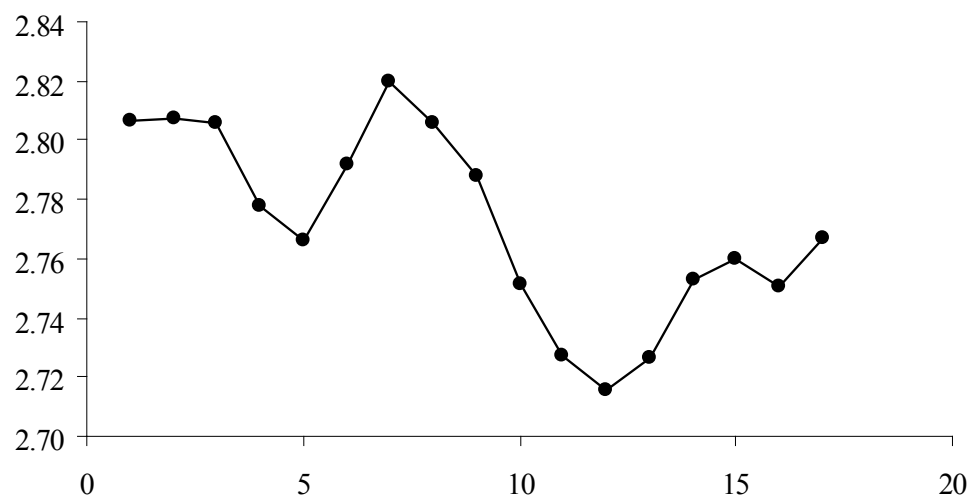

Fig. 10. The extrapolated rate for the day $17\left(\right.$ July $\left.11^{\text {th }}\right)$ using the MHR method with 16 nodes and the weighted mean value (16)

Source: own study.

The true rate of US dollar on July $11^{\text {th }}$ is 2.8123 (Figure 11). 


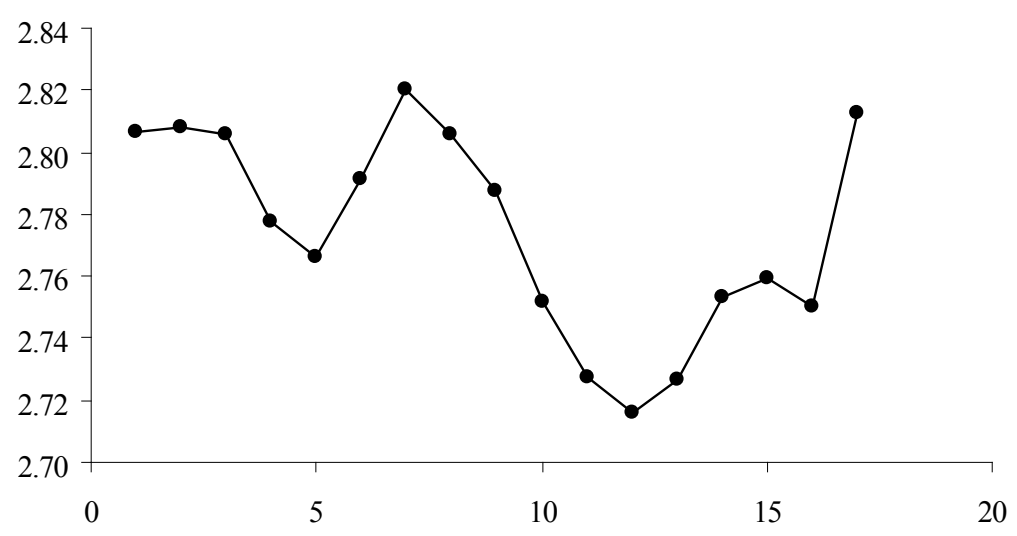

Fig. 11. The true rate of US dollar for the day 17 (July $11^{\text {th }}$ )

Source: own study.

In the author's opinion, the extrapolated values of 2.7650 (15) and 2.7672 (16) and the extrapolated rates in example 1 preserve the increasing trend and they are good enough to be one of the factors for making a decision of buying or selling the currency. The anticipated values, calculated by the MHR method, are applied in the process of decision making: to follow the action or not, to do one thing or another. The extrapolated values can be used to make a decision in many branches of science and economics.

\section{Conclusions}

The method of Hurwitz-Radon Matrices leads to curve interpolation and value extrapolation depending on the number and location of data points. No characteristic features of a curve are important in the MHR method: failing to be differentiable at any point, the Runge's phenomenon or differences from the shape of polynomials. These features are very significant for classical polynomial interpolations and extrapolations. The MHR method gives the possibility of reconstructing a curve and anticipating the data points. The only condition is to have a set of nodes according to assumptions in the MHR method. Data representation and curve extrapolation by means of the MHR method is connected with possibility of changing the nodes coordinates and the reconstruction of new data or curve for a new set of nodes. The same MHR interpolation and extrapolation is valid for discrete and continuous data. Main features of the MHR method are: the accuracy of data reconstruction depending on the number of nodes; interpolation or extrapolation of a curve consisting of $L$ points is connected with the 
computational cost of rank $O(L)$; the MHR method is dealing with local operators: the average OHR operators are built by successive 4,8 or 16 data points, what is connected with smaller computational costs than using all nodes; the MHR is not an affine interpolation ${ }^{22}$.

Future works are connected with: the possibility to apply the MHR method to threedimensional curves ( $3 \mathrm{D}$ data), computing the extrapolation error, object recognition ${ }^{23}$ and the MHR version for equidistance nodes.

\section{Notes}

1 Brachman, Levesque (2004).

2 Fagin, Halpern, Moses, Vardi (1995).

3 Straffin (1993).

4 Watson (2002).

5 Markman (1998).

6 Sowa (2000).

7 Soussen, Mohammad-Djafari (2004).

8 Tang (2005).

9 Kozera (2004).

${ }^{10}$ Dahlquist, Bjoerck (1974).

${ }^{11}$ Ralston (1965).

12 Eckmann (1999).

13 Citko, Jakóbczak, Sieńko (2005).

${ }^{14}$ Tarokh, Jafarkhani, Calderbank (1999).

${ }^{15}$ Sieńko, Citko, Wilamowski (2002).

${ }^{16}$ Sieńko, Citko (2002).

17 Jakóbczak (2007).

18 Jakóbczak, Shape Representation and Shape Coefficients... (2010).

19 Jakóbczak (2009).

${ }^{20}$ Jakóbczak, Application of Hurwitz-Radon Matrices... (2010).

${ }^{21}$ Jakóbczak, Object Modeling Using Method of Hurwitz-Radon Matrices... (2010).

22 Jakóbczak, Implementation of Hurwitz-Radon Matrices... (2010).

${ }^{23}$ Jakóbczak (2011).

\section{References}

Brachman, R.J., Levesque, H.J. (2004). Knowledge Representation and Reasoning. Morgan Kaufman, San Francisco. 
Citko, W., Jakóbczak, D., Sieńko, W. (2005). On Hurwitz-Radon Matrices Based Signal Processing. Workshop Signal Processing at Poznan University of Technology.

Dahlquist, G., Bjoerck, A. (1974). Numerical Methods. New York: Prentice Hall.

Eckmann, B. (1999). Topology, Algebra, Analysis - Relations and Missing Links. Notices of the American Mathematical Society 5(46), 520-527.

Fagin, R., Halpern, J.Y., Moses, Y., Vardi, M.Y. (1995). Reasoning About Knowledge. MIT Press.

Jakóbczak, D. (2010). Application of Hurwitz-Radon Matrices in Shape Representation. In: Banaszak, Z., Świć, A. (eds.) Applied Computer Science: Modelling of Production Processes 1(6), 63-74. Lublin: Lublin University of Technology Press.

Jakóbczak, D. (2009). Curve Interpolation Using Hurwitz-Radon Matrices. Polish Journal of Environmental Studies 3B(18), 126-130.

Jakóbczak, D. (2010). Implementation of Hurwitz-Radon Matrices in Shape Representation. In: Choraś, R.S. (ed.) Advances in Intelligent and Soft Computing 84, Image Processing and Communications: Challenges 2, 39-50. Springer-Verlag, Berlin Heidelberg.

Jakóbczak, D. (2010). Object Modeling Using Method of Hurwitz-Radon Matrices of Rank k. In: Wolski, W., Borawski, M. (eds.) Computer Graphics: Selected Issues, 79-90. Szczecin: University of Szczecin Press.

Jakóbczak, D. (2011). Object Recognition via Contour Points Reconstruction Using HurwitzRadon Matrices. In: Józefczyk, J., Orski, D. (eds.) Knowledge-Based Intelligent System Advancements: Systemic and Cybernetic Approaches, 87-107. IGI Global, Hershey PA, USA.

Jakóbczak, D. (2010). Shape Representation and Shape Coefficients via Method of Hurwitz-Radon Matrices. Lecture Notes in Computer Science 6374 (Computer Vision and Graphics: Proc. ICCVG 2010, Part I), Springer-Verlag Berlin: Heidelberg, 411-419.

Jakóbczak, D. (2007). 2D and 3D Image Modeling Using Hurwitz-Radon Matrices. Polish Journal of Environmental Studies 4A(16), 104-107.

Kozera, R. (2004). Curve Modeling via Interpolation Based on Multidimensional Reduced Data. Silesian University of Technology Press, Gliwice.

Markman, A.B. (1998). Knowledge Representation. Lawrence Erlbaum Associates.

Ralston, A. (1965). A First Course in Numerical Analysis. New York: McGraw-Hill Book Company.

Sieńko, W., Citko, W. (2002). Hamiltonian Neural Net Based Signal Processing. The International Conference on Signal and Electronic System ICSES.

Sieńko, W., Citko, W., Wilamowski, B. (2002). Hamiltonian Neural Nets as a Universal Signal Processor. $28^{\text {th }}$ Annual Conference of the IEEE Industrial Electronics Society IECON. 
Sowa, J.F. (2000). Knowledge Representation: Logical, Philosophical and Computational Foundations. Brooks/Cole, New York.

Soussen, C., Mohammad-Djafari, A. (2004). Polygonal and Polyhedral Contour Reconstruction in Computed Tomography. IEEE Transactions on Image Processing 11(13), 1507-1523.

Straffin, P.D. (1993). Game Theory and Strategy. Mathematical Association of America, Washington, D.C.

Tang, K. (2005). Geometric Optimization Algorithms in Manufacturing. Computer-Aided Design \& Applications 2(6), 747-757.

Tarokh, V., Jafarkhani, H., Calderbank, R. (1999). Space-Time Block Codes from Orthogonal Designs. IEEE Transactions on Information Theory 5(45), 1456-1467.

Watson, J. (2002). Strategy - An Introduction to Game Theory. University of California, San Diego. 